\title{
Autolesiones sin intención suicida presente en adolescentes
}

\section{Self-inflicted injuries present in adolescents caused without suicidal intention}

\author{
Jessica Vanessa Quito Calle ${ }^{1}$ y Jenny Mariela Reinoso Brito $^{2 *}$ \\ ${ }^{1}$ Universidad de Cuenca \\ ${ }^{2}$ Universidad Católica de Cuenca \\ *jeereinosobrito@gmail.com
}

DOI: https://doi.org/10.26871/killkana_salud.v3i2.234

\begin{abstract}
Resumen
Contexto: En esta investigación se examinaron las características sociodemográficas, los niveles y tipos de autolesiones sin intención suicida más frecuentes en adolescentes. Objetivo: describir las características socio-demográficas de los adolescentes de la comunidad terapéutica "Hogar Crecer", de la ciudad de Cuenca, así como los niveles y tipos de conductas autolesivas. Procedimiento: El estudio tiene una modalidad cuantitativa, donde participaron 40 adolescentes internos de la comunidad terapéutica "Hogar Crecer" de la ciudad de Cuenca, de sexo masculino, con una edad comprendida entre 13 y 17 años, quienes respondieron al test Cédula de Autolesión. Resultados: Para el análisis de resultados se interpretó de la siguiente forma: 0-1: ausencia de autolesión, 2-10: autolesión leve, 11-19: autolesión moderada, 20-25: autolesión grave. Presentando un alto nivel de efectividad con un alfa de Cronbach de 0,80 . Conclusiones: se observa que el nivel de autolesión moderado aparece en el 32,5\% de adolescentes de 16 años, en el $60 \%$ que cursa la secundaria y el 62,5\% que pertenece a la zona rural. Referente a los tipos de autolesión el más frecuente es cortarse hasta sangrar la piel con el $85 \%$; el 77,50\% prefiere rayar, marcar, pinchar la piel sin sangrar; el 72,50\% se estrella la cabeza con algún objeto o contra la pared y el $65 \%$ frota su piel con objetos punzantes, vidrios o fichas.
\end{abstract}

Palabras clave: Autolesiones, intención suicida, adolescentes.

\begin{abstract}
Introduction: in this research, the most common socio-demographic characteristics, levels, and types of self-injuries caused without suicidal intention in adolescents were analyzed. Objective: To describe the socio-demographic characteristics of the teenagers at "Hogar Crecer", a therapeutic community in the city of Cuenca, as well as the types and levels of their self-injurious behavior. Procedure: The study follows a quantitative methodology, where 40 male adolescents, aged between 13 and 17 residents of "Hogar Crecer", participated and completed the 'Self-injurious Test Form'. Results: The results were interpreted as follows: 0-1: there is a lack of self-inflicted injuries, 2-10: minor self-inflicted injuries, 11-19: moderate self-inflicted injuries, and 20-25: serious self-inflicted injuries presenting a high-effectiveness level with a Cronbach alpha of 0.80. Conclusions: It has been concluded that a moderate level of self-inflicted injuries was observed in $32.5 \%$ in 16-year-old adolescents, $60 \%$ in secondary school students, and $62.5 \%$ in rural areas inhabitants. With regard to the types of self-inflicted injuries, the most frequent is cutting the skin to the point of bleeding with $85 \% ; 77,50 \%$ prefer scratching, marking, or puncturing their skin without causing bleeding; 72,50\% hit their heads against some kind of object or a wall, and 65\% rub their skin using sharp objects, glasses, or small game pieces.
\end{abstract}

Keywords: self-injuries, suicidal intention, adolescents.

\section{Introducción}

El Manual Diagnóstico y Estadístico de los Trastornos Mentales(DSM-5), define a las autolesiones sin intención suicida como una conducta mediante la cual el individuo se realiza de forma intencional lesiones en la superficie de su cuerpo produciendo sangrado, hematoma o dolor, con la seguridad de que la lesión solo conllevará a un daño físico leve o moderado. Se realiza con la expectativa de aliviar un estado cognitivo negativo, para solucionar una dificultad interpersonal, e inducir un estado de sentimientos positivos. $^{1}$

Un estudio realizado en Australia reportó que el 8,1\% de la población general se ha autolesionado alguna vez a lo largo de su vida. En estudios de revisión se puede observar que los adolescentes tienen prevalencias que van del 13 al $23,2 \%$ y la población que ha requerido hospitalización ha reportado un incremento de esta problemática. ${ }^{2}$ 
Según estudios comunitarios realizados en la población general, se muestra que casi del 13 al $29 \%$ de los adolescentes del 4 al $6 \%$ de la población adulta han recurrido a conductas autolesivas al menos una vez en su vida. De acuerdo a este mismo estudio, se indica que la edad de inicio de estas conductas está entre los 10 y 15 años. A lo largo del tiempo se ha descrito que las conductas autolesivas son más frecuentes en las mujeres adolescentes. ${ }^{3}$

El presente estudio se realizó con el objetivo de describir las características sociodemográficas de los adolescentes de la comunidad terapéutica "Hogar Crecer" de la ciudad de Cuenca, así como los niveles y tipos de conductas autolesivas presentes en esta población a lo largo del tiempo. Para cumplir con estos objetivos se aplicó el test Cédula de Autolesión (CAL), a una muestra de 40 adolescentes internos de la comunidad terapéutica "Hogar Crecer" desde el mes de septiembre del año 2017 a enero del 2018.

La psicología ha mostrado preocupación sobre el tema de autolesión que está implicada en especial con adolescentes, por ello se han realizado varias investigaciones a nivel mundial, que evidencian los principales aspectos de esta problemática, la misma que aumenta de modo significativo. No obstante, la mayoría de las investigaciones están focalizadas a poblaciones clínicas, dando a conocer relaciones existentes de esta conducta con trastornos psiquiátricos. ${ }^{4}$ Por lo antes expuesto es que esta investigación cobra relevancia, ya que se aborda la problemática con énfasis en la población adolescente no clínica.

\section{Marco teórico o antecedentes}

Si bien en la actualidad se han considerado a los factores sociales como los favorecedores de un incremento de la prevalencia de las autolesiones, existen referencias en donde muestran que las autolesiones son un fenómeno conocido por la comunidad psiquiátrica desde hace casi 90 años. Offer y Bargglow en 1960, fueron los primeros en definir el cortarse las muñecas en relación al síntoma en adolecentes como un fenómeno en hospitales psiquiátricos. ${ }^{5}$

La adolescencia es considerada como una etapa vulnerable del ser humano, debido a los cambios tanto físicos como psicológicos. Uno de los aspectos más importantes es el desarrollo social, en donde son significativos tanto las relaciones interpersonales como el contexto del menor. Una de las principales preocupaciones en esta etapa es la constante búsqueda de aceptación en su grupo de pares con quienes crea un mayor apego, desplazando el mismo hacia sus padres. Es por ello que las autolesiones pueden ser predominantes en la adolescencia, porque puede estar relacionado con la búsqueda de identidad y aprobación. ${ }^{6}$

Las autolesiones sin intención suicida se definen como cualquier tipo de conducta intencional y autodirigida, que causa la ruptura de tejidos corporales. Esta conducta tiene varias manifestaciones; entre ellas: los cortes, quemaduras y golpes. Desde un punto de vista clínico, cortarse supondría un desahogo emocional, búsqueda de regulación afectiva, control de impulsos o llamar la atención de otros.
Entre los factores más influyentes están la presencia de psicopatología en ellos o sus familiares, disfunción familiar, presencia de adversidad social o la presencia de eventos estresantes recurrentes. ${ }^{7}$

Según la literatura, se encuentran varios conceptos para referirse a las conductas autolesivas. Entre ellos: autolesión, conducta autolesiva, autoagresión deliberada (equivalentes a los términos en inglés self injury, self-injurios behavior, self harm y deliberate self harm, respectivamente). Además, el término es acuñado recientemente en la quinta edición del Manual Diagnóstico y Estadístico de los Trastornos Mentales, como una nueva categoría dentro de los diagnósticos: autolesiones no suicidas, con su término en inglés: Non-suicidal self-injury (NSSI). ${ }^{8}$

A continuación, se muestran los criterios más relevantes del manual DSM-5, para diagnosticar el trastorno de autolesiones sin intención suicida:

a) En el último año, durante al menos 5 días, la persona se ha autoagredido de manera intencional causando daño en su cuerpo, produciendo sangrado, moretones o dolor, como por ejemplo: cortarse, golpearse, quemarse, frotarse excesivamente con propósitos de que la lesión cause solamente un daño físico, leve o moderado.

b) La lesión provocada intencionalmente se asocia por lo menos con dos de los siguientes ítems:

1. Pensamientos o sentimientos dañinos, como depresión, ansiedad, tensión, enojo, angustia generalizada o autocrítica, que ocurran en el periodo inmediatamente antes de la conducta autolesiva.

2. Antes de incidir en el acto, se genera un periodo de intranquilidad acerca de la conducta a realizar que es difícil de oponer resistencia.

3. El impulso para llevar a cabo la autolesión ocurre frecuentemente.

4. La acción tiene un propósito, alivio de un sentimiento y/o estado cognitivo negativo, conflicto interpersonal, o provocar un estado emocional positivo. El paciente predice que este acto va a ocurrir durante o después de la autolesión.

c) La conducta adquiere consecuencias, afectando su entorno interpersonal, académico, entre otros.

d) La conducta no ocurre únicamente en estados de psicosis, delirio o intoxicación. En personas que presentan el trastorno del desarrollo, la conducta no es parte de un patrón de estereotipos repetitivos. ${ }^{9}$

No existen causas específicas por las cuales una persona se autolesiona; por lo general lo hacen como resultado de una incapacidad para afrontar un sufrimiento psicológico, debido a que le resulta difícil regular, expresar o entender sus emociones. Es por ello que entre los objetivos que consiguen al realizar esta conducta están: lograr una sensación de alivio, generar una distracción frente a las emociones dolorosas, tener un control sobre su propio cuerpo, sentir dolor físico para llenar un vacío emocional, exteriorizar sus 
sentimientos internos, comunicar sus sentimientos de angustia al mundo exterior y como castigo. ${ }^{10}$

Es importante también hablar acerca de los factores de riesgo que aumentan la probabilidad de que un individuo sufra una lesión. Con respecto a las características personales están la baja autoestima, la escasa capacidad para solucionar problemas, el alto nivel de impulsividad, ira y hostilidad. Otro de los factores son los trastornos psiquiátricos, en especial la depresión, el trastorno de conducta y patología dual asociado al consumo de sustancias psicoactivas. Por último las características familiares, convivir con un solo progenitor, problemas de convivencia entre los padres y escaza calidez en las relaciones familiares. ${ }^{11}$

En la misma línea, los factores de protección son importantes para la prevención. Entre los principales están los factores individuales como la confianza en sí mismo, buenas habilidades sociales, búsqueda de ayuda cuando atraviesan dificultades y para la toma de decisiones importantes, receptividad ante experiencias de otras personas y capacidad de trascendencia. Los factores familiares juegan un papel muy importante en los adolescentes cuando existe una buena relación y apoyo entre los miembros de la familia. También los factores culturales como la integración social, buenas relaciones con sus compañeros, profesores y otros adultos. ${ }^{12}$

Ahora bien, con respecto a las consecuencias de las autolesiones se tiene, que se intensifiquen los sentimientos de vergüenza, culpa y baja autoestima, complicaciones e infecciones en las heridas, cicatrices permanentes, lesiones graves y el empeoramiento de los problemas y trastornos no diagnosticados si no se tratan de un modo adecuado. ${ }^{13}$

\section{Metodología}

Para realizar la investigación se solicitó a los adolescentes y a los padres de familia la firma del consentimiento informado respectivo. El estudio realizado se basó en una investigación de campo, que se efectuó en la comunidad terapéutica "Hogar Crecer" de la ciudad de Cuenca. Es de tipo cuantitativa, exploratoria y descriptiva. La muestra es de 40 adolescentes con un rango de edad entre 13 a 17 años.

La tabulación de los resultados fue realizada por medio del programa de representación de datos SPSS Statistics. El reactivo fue la Cédula de Autolesión (CAL), que es un cuestionario que consta de 13 ítems planteados para identificar y calcular las autolesiones sin intención suicida. Se tienen ítems del 1 al 12 que pertenecen a los tipos de autolesión. Existe un último ítem que indica la edad de inicio de esta conducta. De los 12 ítems, 5 son los tomados en cuenta dentro de la calificación, misma que va sujeta al número de veces que lo realizó. Para el análisis de los resultados se interpreta de la siguiente forma: 0-1: Ausencia de autolesión, 2-10: Autolesión leve, 11-19: Autolesión Moderada, 20-25: Autolesión grave. Se presentó un alfa de Cronbach de $0,80 .{ }^{14}$

\section{Resultados}

A continuación, se resalta la asociación entre las características sociodemográficas y los niveles de autolesión sin intensión suicida.

Tabla 1. Tabla cruzada: características sociodemográficas y niveles de autolesión sin intención suicida

\begin{tabular}{|c|c|c|c|c|c|c|c|c|c|}
\hline \multirow{2}{*}{\multicolumn{2}{|c|}{$\begin{array}{c}\text { Características } \\
\text { sociodemográficas }\end{array}$}} & \multicolumn{6}{|c|}{ NIVELES } & & \\
\hline & & \multicolumn{2}{|c|}{ Ausencia de autolesión } & \multicolumn{2}{|c|}{ Autolesión leve } & \multicolumn{2}{|c|}{ Autolesión moderada } & \multicolumn{2}{|c|}{ Total } \\
\hline & & $\mathrm{F}$. & P. & $\mathrm{F}$ & P. & F. & P. & $\mathrm{F}$. & P. \\
\hline \multirow[t]{6}{*}{ Edad } & 13 años & 1 & 2,5 & 0 & 0 & 0 & 0 & 1 & 2,5 \\
\hline & 14 años & 1 & 2,5 & 2 & 5 & 0 & 0 & 3 & 7,5 \\
\hline & 15 años & 0 & 0 & 12 & 30 & 0 & 0 & 12 & 30 \\
\hline & 16 años & 0 & 0 & 0 & 0 & 13 & 32,5 & 13 & 32,5 \\
\hline & 17 años & 0 & 0 & 0 & 0 & 11 & 27,5 & 11 & 27,5 \\
\hline & Total & 2 & 5 & 14 & 35 & 24 & 60 & 40 & 100 \\
\hline \multirow[t]{4}{*}{ Escolaridad } & Primaria & 2 & 5 & 0 & 0 & 0 & 0 & 2 & 5 \\
\hline & Secundaria & 0 & 0 & 13 & 32,5 & 24 & 60 & 37 & 92,5 \\
\hline & Ninguno & 0 & 0 & 1 & 2,5 & 0 & 0 & 1 & 2,5 \\
\hline & Total & 2 & 5 & 14 & 35 & 24 & 60 & 40 & 100 \\
\hline \multirow[t]{3}{*}{ Ubicación } & Urbana & 2 & 5 & 13 & 32,5 & 0 & 0 & 15 & 37,5 \\
\hline & Rural & 0 & 0 & 1 & 2,5 & 24 & 60 & 25 & 62,5 \\
\hline & Total & 2 & 5 & 14 & 35 & 24 & 60 & 40 & 100 \\
\hline
\end{tabular}


La asociación de las características sociodemográficas con los niveles de autolesión sin intención suicida, se observa que el $32,5 \%$ presenta un nivel de autolesión moderada a los 16 años. Así mismo al asociar la variable de escolaridad con los niveles de autolesión, se puede observar que un $60 \%$ de adolescentes que han cursado la secundaria presentan un nivel de autolesión moderada y el $32,5 \%$ presentan autolesión leve. De la misma forma, en la asociación de la variable ubicación con los niveles de autolesión, se puede evidenciar que el $60 \%$ que pertenece a la zona rural presenta un nivel moderado de autolesión.

Por consiguiente, se evidencian los tipos de autolesiones más utilizados por los adolescentes:

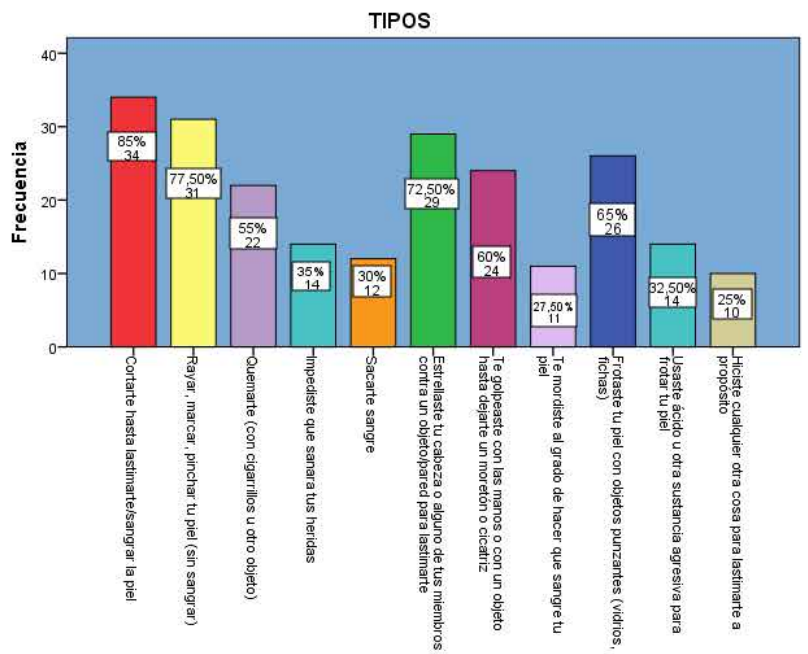

Fig. 1. Tipos de autolesiones

Con respecto a los tipos de autolesión, se observa que el $85 \%$ de adolescentes se ha cortado hasta sangrar la piel. Las otras formas más frecuentes de autolesión son rayar, marcar, pinchar la piel sin sangrar con un 77,50\%; estrellarse la cabeza contra un objeto o pared con un 72,50\%; frotar su piel con objetos punzantes, vidrios o fichas corresponde a un $65 \%$; golpearse con las manos o con un objeto hasta dejar un moretón o cicatriz con un $60 \%$ y quemarse con cigarrillos $\mathrm{u}$ otros objetos en un $55 \%$. En un porcentaje menor se encuentra impedir que sanen sus heridas y usar ácido u otra sustancia agresiva para frotar la piel.

\section{Conclusiones y recomendaciones}

El presente estudio tuvo como finalidad el contribuir a la teoría actual de las autolesiones sin intención suicida en adolescentes, dada la carencia de datos al respecto en el Ecuador. Los resultados confirmaron la hipótesis planteada, mostrando que los adolescentes presentan un nivel moderado de autolesión. Es necesario elaborar propuestas psicoeducativas que podrían producir una disminución de la frecuencia de autolesiones en esta población.

Con respecto a la edad en la que se ha presentado con mayor frecuencia las autolesiones sin intención suicida, ha sido entre 15 y 17 años, siendo un grupo mayoritario los adolescentes que tienen 16 años de edad. Esto concuerda con un estudio realizado por Romero en México en el año 2013 en donde los adolescentes que se autolesionan tienen entre 12 y 17 años. ${ }^{15}$ De acuerdo a otra investigación realizada en Perú por Alvino et al. en el año 2016, explica que la edad en la que empiezan estas conductas oscila entre los 12 y 16 años. ${ }^{16}$ Así mismo un estudio realizado por Bustillos en Bolívar en el año 2014, menciona que los adolescentes de 10 a 14 años son los más propensos a las autolesiones. ${ }^{17}$ De la misma manera en un estudio realizado por Mollá en España en el año 2015, se puede evidenciar que la edad más frecuente de inicio de autolesiones se sitúa entre los 11 y 15 años. $^{18}$

Según Vázquez en una investigación realizada en Perú en el año 2009, indica que las conductas autolesivas inician en la etapa de la adolescencia o pubertad. ${ }^{19}$ Del mismo modo una investigación realizada por Silva et al. en México en el año 2014 muestra que en países como Estados Unidos, Canadá y Europa el fenómeno de autolesión está presente en los adolescentes. ${ }^{20}$ Es por ello que se debe tomar en cuenta que esta población al estar en una etapa vulnerable a cambios típicos de su edad, es más propensa a recurrir a este tipo de conductas.

En la misma línea de acuerdo al sexo de los participantes, la totalidad de la muestra corresponde al sexo masculino. La afirmación anterior coincide coincidiendo con los trabajos de Rospigliosi en Perú en el año 2011, en donde se observa que los hombres se han autolesionado en mayor porcentaje que las mujeres. Así mismo otro estudio realizado en Lisboa por Guerreiro en 2014, muestra que las mujeres se autolesionan con mayor frecuencia que los hombres, siendo distinto a esta investigación. ${ }^{21}$

Según Díaz en Perú en el año 2015, indica que existe mayor preocupación de prácticas de autolesiones en adolescentes de zonas rurales. ${ }^{22}$ Esta investigación concuerda con los resultados obtenidos en la investigación, en donde los adolescentes que pertenecían al sector rural eran más propensos a autolesionarse.

Ahora bien, el nivel moderado de autolesión sin intensión suicida es el más frecuente en los adolescentes de la muestra, estos datos concuerdan con Roca et al. en Barcelona en el año 2012, quien reporta que la mayor parte de sus participantes había mostrado autolesiones moderadas o severas. ${ }^{23}$ En una investigación realizada en Ecuador por Mazón en el año 2017, se puede evidenciar que los adolescentes muestran un nivel de autolesión grave, lo cual demuestra que son muy pocos los estudios realizados en base a lo que corresponden los niveles de autolesión.

Por otra parte, los tipos de autolesión a los que recurren los adolescentes con mayor frecuencia son los cortes hasta hacer sangrar la piel; el rayar, marcar, pinchar la piel sin sangrar; estrellarse la cabeza contra la pared o un objeto; frotar la piel con objetos punzantes y golpearse hasta hacerse un moretón o cicatriz. Esto es corroborado por un estudio realizado por Muñoz et al. en Colombia en el año 2016, en 
donde se observa que los tipos más frecuentes de autolesiones son los cortes superficiales en muñecas y antebrazos, así como los golpes y rasguños. ${ }^{7}$ Otro estudio realizado por Marín en México en el año 2013, menciona que el tipo de autolesión más significativo es cortarse, seguido de frotarse con objetos cortantes y el menos significativo rayar y marcar la piel. ${ }^{24}$ Así mismo en una investigación realizada por Neira en Madrid en el año 2014, muestra que el método más frecuente empleado por los adolescentes es golpearse a sí mismo y realizarse cortes, todos ellos se autolesionaron de una manera impulsiva. ${ }^{25}$ En la misma línea según un estudio realizado por Mazón en Ecuador en el año 2015, muestra que los métodos más utilizados para autolesionarse son los cortes, intoxicación y uso de pastillas, ${ }^{26}$ mostrando que existen nuevos métodos de autolesión que no han sido nombrados en esta investigación.

\section{Fuente de Financiamiento}

Este estudio es autofinanciado.

\section{Conflicto de Intereses}

No existen conflictos personales, profesionales, financieroso de otro tipo.

\section{Consentimiento Informado}

Los autores cuentan con el consentimiento informado de los pacientes para la investigación, la publicación del caso y sus imágenes.

\section{Referencias Bibliográficas}

1. Morales JC. Autolesión no suicida en adolescentes peruanas: Una aproximación diagnóstica y psicopatológica. Revista de Neuro-Psiquiatria. 2014;77(4):226. Available from: http://www.upch.edu.pe/vrinve/dugic/revistas/ index.php/RNP/article/view/2192.

2. Flores REU, Hernández CC, Navarrete KP, Figueroa GV. Frecuencia de autolesiones y características clínicas asociadas en adolescentes que ocudieron a un hospital psiquiátrico infantil. Salud Mental. 2013;36(5):417420. Available from: https://liverpool.idm.oclc.org/ login?url=http://search.ebscohost.com/login.aspx?direct= true $\& \mathrm{db}=$ psyh $\& A N=2013-43679-009 \&$ site $=$ ehostlive \&scope=site\%5Cnhttp://eulloa@hotmail.com.

3. Villarroel JG, Jerez SC, Angélica Montenegro MM, Montes CA, Igor MM, Silva HI, et al. Conductas autolesivas no suicidas en la práctica clínica. Primera parte: conceptualización y diagnóstico Non suicidal self injuries in clinical practice. Rev Chil Neuro-Psiquiat. 2013;51(1):3845. Available from: http://www.scielo.cl/scielo.php? script=sci_arttext\&pid=S0717-92272013000100006.

4. Diego J, Salazar E. Autolesiones: Las huellas de lo psicológico en la piel. Departamento de Psicologia Mdrid. 2017;p. 70-72. Available from: http://200.24.17.74: 8080/jspui/bitstream/fcsh/1007/1/TrujilloMonica_2017_ AutolesionesHuellasPsicologico.pdf.
5. Dopico González. El daño autolesiones y comportamientos autodestructivos. Revista Oficial de la sección de Psicología de la Salud de COPG. 2017;p. 45-46. Available from: https://copgalicia.gal/system/files/PDFs/ publicacions/anuario_numero_10_o_dano.pdf.

6. Mendoza VG, Tezón M. Relación entre la observación de contenidos en internet sobre autolesiones respecto a la ejecución de Cutting en una adolescente con Trastorno Depresivo Persistente. 2016;p. 10-15. Available from: http://bibliotecadigital.usb.edu.co/bitstream/10819/3845/ 1/Relaciónentrelaobservación_VivianaGonzálezM_2016. pdf.

7. Mariana L, Henao H, Pineda DS. Generalidades en la evaluación clínica psicológica referentes a las autolesiones no suicidas en adolescentes. Psyconex. 2016;8:1-11. Available from: https://aprendeenlinea.udea.edu.co/revistas/ index.php/Psyconex/article/view/326997/20784220.

8. González Suárez LF, Vasco Hurtado IC, Nieto Betancurt L. Revisión de la literatura sobre el papel del afrontamiento en las autolesiones no suicidas en adolescentes. Cuadernos Hispanoamericanos de Psicología. 2016;16(1):41-56. Available from: https://dialnet.unirioja.es/descarga/articulo/5855292. pdf\%0Ahttps://dialnet.unirioja.es/servlet/extart?codigo= 5855292.

9. Kupfer D. Manual diagnóstico y estadístico de los trastornos mentales. 5th ed. Susan Schultz EK, editor. ESPAÑA; 2014. Available from: https://es.slideshare.net/Josefbg/dsm-5-manualdiagnstico-y-estadistico-de-los-trastornos-mentales.

10. Daniel P, Rodríguez A. La autolesión: Posibles causas y consecuencias, y su manejo exitoso. 2015;p. 50. Available from: http://www.uptc.edu.co/export/sites/default/ eventos/2015/cf/ccp/doc/conf_paulo_acero.pdf.

1. Arciniegas Luzuriaga XF. Autolesiones sin intención suicida en alumnos de un colegio fiscal de la ciudad de Cuenca. Revista Médica HJCA. 2016;8(1):35-39. Available from: http://revistamedicahjca.med.ec/ojs/index.php/ RevHJCA/article/view/66.

12. Bazan Villavicencio DM, Tapia M M. Factores de riesgo en las conductas autodestructivas en adolescentes de 10 a 13 años. 2011;p. 15-30. Available from: http://dspace. ucuenca.edu.ec/handle/123456789/2217.

13. Rodríguez P. Autolesión. 2015;p. 10. Available from: http://www.davinci.edu.co/documents/psicologia/media/ autolesion.pdf.

4. Albores-Gallo L, Méndez-Santos JL, Xóchitl-García Luna A, Delgadillo-González Y, Chávez-Flores CI, Martínez OL. Autolesiones sin intención suicida en una muestra de niños y adolescentes de la ciudad de México. Actas Españolas de Psiquiatría. 2014;42(4):159-168. Available from: http://actaspsiquiatria.es/repositorio/16/90/ESP/1690-ESP-159-168-346514.pdf.

5. Romero M. Adolescentes con autolesiones e ideación suicida: un grupo con mayor comorbilidad y adversidad psicosocial. 2013;122(1):38-39. 
16. Alvino I. Inteligencia Emocional en estudiantes que se autolesionan, de nivel secundario en la I.E.P. Gelicich del Distrito de Tambo - 2015. 2016;p. 76. Available from: http://repositorio.continental.edu.pe/handle/23. continental/3375.

17. Bustillos D. El fenómeno cutting y el estado psicoemocional en las y los adolescentes de 10 a14 años de la escuela Bartoloméde las Casas. comunidad Gradas. Cantón Guaranda. Provincia Bolívar. Periodo 2013. Departamento de 24. Postgrado, Universidad de Bolívar. 2014;p. 79. Available from: http://dspace.ueb.edu.ec/bitstream/123456789/152/ 3/TESINA.pdf.

18. Mollà L, Vila SB, Treen D, López J, Sanz N, Martín LM, et al. Autolesiones no suicidas en adolescentes: Revisión de los tratamientos psicológicos. Revista de Psicopatologia y Psicologia Clinica. 2015;20(1):51-61.

19. Carlos Vásquez. Alexitimia y autolesiones en un 26. grupo de escolares de Lima Metropolitana. Test. 2009;p. 1-125. Available from: http://tesis.pucp. edu.pe/repositorio/bitstream/handle/123456789/411/ VASQUEZ_CONTRERAS_CARLOS_ALEXITIMIA_ ESCOLARES.pdf?sequence $=1 \&$ is Allowed $=\mathrm{y}$.

20. Thyssen LS, Camp IV. Non-Suicidal Self-Injury in Latin America. 2014;37(2):153-157. Available from: http://www.scielo.org.mx/scielo.php?script=sci_arttext\& pid=S0185-33252014000200009.

21. Guerreiro D. Comportamentos autolesivos en adolescentes: características epidemiológicas y análisis de fatores psicopatológicos, temperamento efetivo y estratégias de coping. 2014;p. 5. Available from: http://repositorio.ul. pt/handle/10451/11457.

22. María Díaz CH. El cutting en los estudiantes de la institución educativa Padre Pio Sarobe-Distrito de Santa Rosa de Ocopa-Provincia de Concepción. Universi- dad Nacional de Entre Ríos Paraná. 2015;1:113. Available from: http://www.ugr.es/\{ \}eutrasoc/guiasdocentes/ antropologia_aplicada.pdf.

. Tutusaus XR, Guàrdia J, Jarne A. Las conductas autolesivas nn el ámbito penitenciario. Una revisión del estado del arte. Papeles del Psicologo. 2012;33(2):116128. Available from: http://www.papelesdelpsicologo.es/ pdf/2100.pdf.

. Marín M. Desarrollo y evaluación de una terapia cognitivo conductual para adolescentes que se autolesionan. Ciencia nueva. 2013;p. 219.

25. Díaz M, Hernando DN. Características psicopatológicas , acontecimientos vitales estresantes y conductas autolesivas suicidas y no suicidas en adolescentes evaluados en salud mental. 2014;Available from: https://repositorio. uam.es/handle/10486/661735.

. Mazón JP. Diseño de intervención de acuerdo al Modelo Integrativo Ecuatoriano en base a la relación entre rasgos de personalidad y conducta autolesiva en adolescentes de Riobamba. 2015;p. 40. Available from: http://dspace. uazuay.edu.ec/handle/datos/4651.

Recibido: 27 de febrero de 2018

Aceptado: 16 de mayo de 2019 